\title{
Varieties of Vagueness, Fuzziness and a few foundational (and ontological) questions
}

\author{
Marco Elio Tabacchi ${ }^{1,3}$ and Settimo Termini ${ }^{1,2}$ \\ ${ }^{1}$ Dipartimento di Matematica e Informatica Università di Palermo (Italy) \\ ${ }^{2}$ European Center for Soft Computing, Mieres (Asturias), Spain \\ ${ }^{3}$ Istituto Nazionale di Ricerche Demopolis (Italy)
}

\begin{abstract}
In this paper we discuss the multifaceted nature of vagueness, the limits of (standard) set theory in dealing with the foundational aspects that a really innovating theory of vagueness should manifest, and the difficulties in outlining the possible features that such a type of new formalism should exhibit in order to be able to deal with such innovative aspects. We shall highlight some aspects of the role that Fuzzy Set Theory (FST) can play in this process.
\end{abstract}

Keywords: Vagueness, fuzziness, language, meaning

\section{Introduction}

Vagueness, under its many names and forms (unsharp predicates, uncertainty, approximate and/or incomplete/inexact reasoning, partial knowledge and incomplete information, approximation, and so on), pervades many scientific theories. As soon as vagueness is introduced in a theory, the need for control and for the design of efficient, quantitative methods to exert such control arises. Oddly, such urge is even more present in the very disciplines that could more easily displace it, namely Computer and Information Sciences.

In this paper we will critically review and briefly discuss the following arguments:

remark 1.1 we are far from an adequate treatment (let alone a theory) of vague predicates, and this notwithstanding the important contributions provided by many current and past approaches;

remark 1.2 an adequate foundation of vague predicates should necessarily cross the boundaries of Zermelo-Fraenkel horizon, that is, until we remain inside the traditional, ontological setting of standard set theory, no progress in this sense is possible.

Language and meaning, two of the pillars of the Special Session this paper is presented to, play a topical role in looking for a rationale to these remark. With their help it is possible to verify how much twentieth century's scientific investigation, in general, and Computer/Information science in particular, have widened the spectrum of problems that can be tackled through the tools offered by scientific methodology

Following a further elaboration of some ideas presented in [1, 2] and also in [3], we suggest that the direction for a meta-critical approach leaving behind the traditional, foundational ontology of present day mathematics is the one that considers strongly constructive aspects, such as the one explored in different contests and for different purposes by Beeson [4] and Sambin [5] (but again see also [2]). How these considerations can be turned into application, however, remains problematic indeed, and to be seen. A suggestion on how to translate these general remarks into feasible procedures can come by the way FST has evolved in its history. The direction of this evolution is clearly in tune with the general approach of both authors mentioned before, and the similarities does not end there. However, the standard formalism of FST is reducible to the standard language of mathematics (see, for instance, [2]). So, when we speak about the role that FST can provide in confronting these problems we refer to two things: the new "visualization" it provides and the various extensions that have been envisaged to go beyond the boundaries of "numerical accuracy" - linguistic variables, computing with words etc. These, however, are far from being completely understood, and only non foundational settings for them is available at present.

\section{Open questions}

In this section we have summarized what we think are the important question that has to be answered in order to foundational theory of vagueness. A synopsis is presented in Fig. 1.

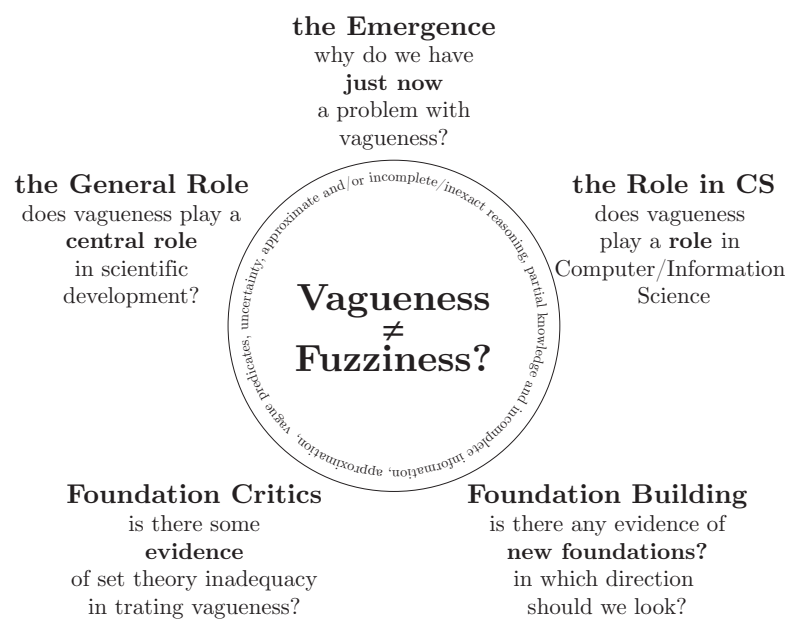

Fig. 1: the questions advanced in this paper.

question 2.1 (the Emergence) Why the problem of vagueness presents to us just now? What changes in our ontologies have forced us to consider something we never considered before? 
question 2.2 (the General Role) Does vagueness plays a role in the present development of scientific investigation? Is the role of vagueness central in scientific development, or just a sort of intellectual, sophisticated caprice?

question 2.3 (the Role in CS) Does vagueness play a specific role in Computer/Information Science?

question 2.4 (the Foundation critics) Is there an evidence of set theory inadequacy in treating vagueness?

question 2.5 (the Foundation building) Are there evidences for a new foundational theory of vagueness? In which direction should we look at?

And, finally, question 2.6 Are fuzziness and vagueness really two different disciplines? In which sense they can be considered as such?

We will discuss these questions in the following sections.

\section{The Emergence}

The idea of vagueness, despite frequent hindering by nomenclature, is ubiquitous in scientific theories. We can call it vague predicates, uncertainty, approximate and/or incomplete/inexact reasoning, partial knowledge and incomplete information, approximation, but we are always referring to concepts that are describing a different aspect or tentative solution for the problem of vagueness. And as soon as vagueness creeps in the discussion, in any of its possible forms and with any of its names, the problem of its control comes along as well like a seemingly unavoidable consequence. The most radical proposal for a practical solution of such problem can be attributed to Frege: eradicating vagueness through strategies of regimentation of the languages for which vagueness itself stands as a problem. The reason for such a radical approach is now a question for historians of mathematics, but as Jean van Heijenoort [6] remarks, Frege "would not have been able to formulate these [logical] laws without leaving vagueness out of the picture." In fact, "the only way to proceed at an early stage in the development of a science is by bold simplification and abstraction". Van Heijenoort's conclusions give some hope for the future: "Frege's disregard of vagueness and other vagaries was, in a way, inevitable. But his logical laws have been formulated more than hundred years ago, and it is now perhaps time to look at the vagaries." (emphasis added)

How can vagueness be acknowledge by logic? Rohit Parikh's [7] analysis of vague predicates is an example and a starting point. In his work Parikh observes that words referring to observational predicates denote vague predicates, and not predicates in Frege's sense. This is not limited to natural languages (where this statement is obvious - as we have seen the reduction operated by Frege is by necessity, not by sheer description) but "affects physics and, in fact, any science that attempts to correlate observation with words or num- bers." Parikh goes along in proving that vague predicates are necessary, since in suitable structures - the socalled observationally connected spaces - it is not possible to define non-trivial observational predicates. From this follows the proposal of an intuitionistic bent Theory of Vague Reals, that can be used only when "measurements can, at least in theory, be arbitrary accurate", and is not applicable when there is "an inherent limit to accuracy" - such as, for instance, in the case of ordinary language. While not yet a Theory of Vague Predicates, and lacking in implementation, Theory of Vague Reals provides useful ideas on how to incorporate vagueness in a formal systems, and indicates a path to follow. It is, then, time to look at the vagaries, and not only in logic, as proposed by van Heijenoort and hinted by Parikh, but in other disciplines as well. While in logic it may seem like a luxury item, in many other fields of enquiry, as formally defined as logic if not more so, vagueness is intrinsic in the definition of the problems, especially when real world limitations such as computational limits or measure problems are involved, and its presence makes things more complex and intricate.

\section{The General Role}

The central role of what we could define as "a lack of precision" (by using - as Middle Ages Theology - negative definitions) is witnessed, implicitly, in all experimental sciences, and explicit acknowledgements not only of its presence, but also of the central role that it can play, can be found more frequently than expected. For instance, the noted structuralist physicist Günther Ludwig [8] maintained that:

One of the most fundamental facts of experimental physics a student has to learn at the beginning of his studies is that no measurement is precise [...] A theoretical physicist will realize that there is another field of imprecision. He will realize that every mathematical theory used as a picture of reality cannot be regarded as a precise picture. He will realize that there is no difference in principle between a socalled exact theory and an approximation picture of reality. What is the meaning of approximation and imprecision in this field?

As another physicist, Lyman G. Parratt [9] stressed, there is a positive side of this feature:

Every fact in Science, every law of nature as devised from observations is intrinsically "openended", i.e. contains some uncertainty and is subject to future improvement.

\section{$[\ldots]$}

The features that distinguishes scientific knowledge is not only that there is a clear recognition of uncertainty but that the degree of uncertainty can usually be rather well determined

We can see how all these aspects are just facets of the same problem, and by agreeing on the fact that vague- 
ness is the unifying notion, the centrality of the vagueness problem speaks for itself.

\section{The Role in CS}

The previous statement is even more actual and cogent in Information systems, and more in general in Computer Science. If we consider the role of scientists as intelligent agents, computer science is the kind of dynamic, continuous environment for which any deliberation, any choice has to be made in a frenetic world of ever-changing behaviors and situations. In such context, we cannot exorcize vagueness and must, somehow, come to terms with its presence, and use it at our advantage.

To look at these problems in the context of Computer and information Sciences, we have to consider the general context in which such new sciences were conceived and the way in which they developed. A very useful tool is provided by the scholarly investigations provided by Seising [10] and Montagnini [11] (but see also [12, 13]). Seising has also specifically studied how "the absence of strict boundaries" has influenced the development of disciplines in the past Century [14].

These developments have restructured the traditional boundaries, in our view, between disciplines, and moreover between hard and soft sciences, indicating analogies between art and technology, showing that the similarities between artifacts, technological devices and products of art can be, for some aspects, stronger than the ones between hard science and technology.

We refer to a few recent papers for an overview of these questions, stressing that this is the general context in which questions related to the search of a true foundation of vagueness and other innovative features of information sciences should be looked for.

\section{Foundation Critics}

According to Pultr [15], Fuzzy Sets cannot model vague predicates in a general sense:

Roughly speaking, we know that fuzzy sets endowed with fuzzy-equivalences can be adequately represented by means of fuzzy-equivalence alone. In the representation the fuzzy sets appear as couples $(\mathrm{X}, \mathrm{Y})$ of crisp subsets of universes with a kind of distance (resulting from the fuzzy equivalencies) and one operates with them crisply.

This result helps understanding whether the theory of fuzzy sets provides a foundation for a notion of generalized membership - or belonging to a degree - which is really innovative and captures some of the peculiar aspects of vagueness. Pultr shows that everything that is done with Fuzzy Sets can be done without the notion of generalized membership function. As such, the theory is enriched with a non trivial representation, but its eventual role as a theory of vagueness is not to be. A parallel between the two instances of generalized characteristic function, the main feature of vague predicates and the basic starting point of fuzzy sets can be of help. In vague predicates, vagueness arises from the fact that there is no automatic, mechanical way to uniformly decide whether a predicate holds or not of a certain individual; in fuzzy sets, there are individuals with definite values of partial belonging. The role of vagueness is not exhausted by assigning degree of membership, be they provided through statistical evaluations as in Max Black's theory of consistency [16] or by any of the many methods published in fuzzy literature. Vagueness reminds us that in order to decide membership additional contextual information must be provided in a non-uniform way. In a way, the situation is similar to the one of the original, simple and intuitive idea of verisimilitude and the impact that the critical results of David Miller [17, 18] had on it. Miller proved that the choice of the meaningful parameters of a certain system is crucial for having a certain ordering of theories which approximate the truth - a result which is devastating for a simple minded view of the way in which theories approach truth. The consequence is that we must look for less elementary ways of facing the crucial problem of verisimilitude, something that we maintain is strictly related to the problem of defining and constructing a general theory of vagueness.

That is not to undermine the notion of membership grade: right visualizations are very important, as they can trigger important and unusual developments of a notion, and we cannot be grateful enough to Lotfi Zadeh $[12,13]$ for having advanced this notion and defended it. But a good visualization - however important for posing a problem in a nonstandard way - is not enough for constructing new innovative, durable theories, if we are not able to provide also a setting in which they are naturally rooted. Notwithstanding the crucial role that the visualization through generalized membership functions played in the developments of the theory of fuzzy sets, we must admit its appointed place is inside the traditional foundations of mathematics, which in turn - does not offer a proper space for the treatment of the really innovative aspects of vague predicates.

\section{Foundation Buildings}

We are now left with the problem of the construction of a satisfactory theory of vague predicates. We are confident that a general answer does not exists - and as a comprehensive rationalization is out of the scope of this paper, we will limit the reasoning to the following remarks.

remark 7.1 the initial effort should be directed toward the conceptual problems. A general indication of the direction to follow is given by the negative results discussed above.

remark 7.2 the static foundational reference scheme should be abandoned in favor of a strongly constructive approach. The analysis of programming languages can be usefully employed to this end; some theoretical remarks by Beeson [4] can also be reinterpreted in connection with some of the connections between vagueness and mathematics put forward by Bečvár [19]. The general considerations outlining the dynamical foundations of mathematics based on constructive ideas by Sambin [20] can provide the general direction. 
Before briefly discussing Beeson considerations, we shall give a look to Alternative Set Theory, developed by Vopenka, Hajek and the logical school of Prague [21]. AST offers an option for vagueness which is absent from ZF foundation: the introduction of semisets, which makes possible a sort of modeling for vague predicates (see [22] and [23] for a detailed parallel between AST and more traditional set theory). While AST is still very limited when it comes to quantitative manipulation of vague predicates, the way the notion of semisets emerges in this theory is relevant to our effort. The relevant point here is the fact that we can have subclasses of a set which are not subsets in a proper, technical, sense: not all the properties of a set are satisfied by them.

Beeson's polymorphic set theory (his own terms) has, in some points, loose parallels with AST, and helps in clarifying some of the mechanism of subclass formation and definition, mechanisms that can be useful in constructing the foundation of a robust, adequate theory of vagueness. Beeson says about the limits of traditional set theory and the NBG paradigm [4]:

In the history of set theory, proper classes go back to Cantor, who recognized that certain "multiplicities" cannot be collected into a "unity". He referred to such multiplicities as "inconsistent sets". It seems to have been von Neumann who first contemplated the consistent use of proper classes. Later formulations were given by Bernays and finally by Gödel. All these theories regard classes as being just like sets except that they are, in von Neumann's picturesque terminology, "too big". That is, "too big" to be a member of another class. In particular, they are viewed as abstract objects like sets, obeying the law of extensionality: two classes are equal if and only if they have the same members.

After having acknowledged the formal correctness of the received view on Set Theory, Beeson asks whether this approach, notwithstanding its formal adequacy and tractability, is also philosophically sounds, affirming:

The process of abstraction that leads to a set from a symbolic representation breaks down on certain symbolic representations. A class is given to us by a symbolic representation, but it has no extension.

And the main reason for that is that "[a] class, then, is a concrete object not an abstract one". To paraphrase what is told in the received view, "It is the extension of a class which is "too big" to exist; or rather, would be too big if it did exist."

What Beeson is also questioning, in our opinion, is the unicity of approach in traditional foundations. We should defend the plurality of approaches, not for ideological reasons, but in order to build adequate foundations for different purposes - different from the ones that the founding fathers established one century ago. What is questioned here is whether the conceptual assumptions which stay behind the proposed traditional view do not admit alternatives: conceptually different viewpoints which in different situations can appear to be more sound than the received ones.

Beeson draws from these observations the following deduction:

It follows that there is no justification for the principle of extensionality for classes in spite of its formal consistency. Nor should we identify a class with a set, if it exist, which has the same members; one is a concrete object, the other is an abstraction.

But we can also look at the question from a different, more interesting and rich point of view, which can also provide indication for the problem of constructing adequate theories of vague predicates:

In this view a proper class is simply a class whose structure is too rich to permit it to be abstracted away, leaving only an extensional shell. The question of its being "too big" is not relevant; size is only one aspect of structure, and not always the most important. Of course, it may be that certain classes are "too big" to be sets; but the assumption that excessive size is the only reason why the abstraction operation might be undefined appears to be completely unjustified.

\section{Beeson's conclusion is that}

[c]lasses may have a more complex structure than can be summed up by telling what sizes they are. If we abstract away everything but the membership structure, we get sets.

These abstractions have served mathematics well, but they may not be the ideal for the foundations of computer science, and they may have led to mistakes in the foundations of mathematics.

We must look for new, different ways of abstracting away [24]; ways that allow the notion of vague predicates to work as well as it informally works in natural languages, and in everyday routine of scientists.

\section{Fuzziness and vagueness}

We shall briefly comment on the point for which fuzziness can be considered a good approximation of the general notion of vagueness. following the analysis done by Terricabras and Trillas in [25], we affirm that fuzziness is really the best way of "approximating" vagueness, as far as we look for "extensional" representations. But we can say also that the very ingenious ways envisaged for escaping the strictures of "numerical accuracy" by taking into account concepts and aspects of natural language are a basic point to search for innovative ways of formalizing "intensional" features carried on by vagueness. This context in not suited for a long and detailed survey of how cognitive sciences have dealt and still are dealing with the problem of an intensional representation of vagueness, but yet a passing remark can be made on how this is an effort that encompass such different cognitive horizon as philosophy and linguistics. In [26], an intensional stance regarding 
vagueness is taken by considering vague intensions as yielding many extensions, and by supposing the existence of non functional relations that may model such vague intensions. The problem of utility failure for vague predicated is then addressed by maintaining that such predicates provide sharp lookalikes of the vague, as such resolving the problem of their full tolerance (and their presumable lack of utility). This solution is only sketched, and does not provide a substantiated logic to support its claims - and as such no foundation on which to build an intensional representation for approximating vagueness. Nevertheless the intuition of putting asides extensions and to consider vague intensions as a proxy can be useful in the sought after formalization.

\section{Conclusions}

In this paper we have tried to look for new directions in designing unconventional ways for capturing the innovative features of vagueness not usually grasped by the usual approaches [27]. We claim that any formalism based on the same ontological basis of standard mathematics is not powerful enough to support the development of a convincing general theory of vagueness. Our view is again supported by van Heijenoort's analysis of Frege [6]:

[W] hat [Frege] does is to introduce an ontology that would allow his new logical laws to function in a way that should be unobstructed and, moreover, as simple as possible He could not have tackled the problem of vagueness at the moment he was introducing these logical laws without extremely complicating his ontological view of the world.

All contemporary formalized systems are based on ontological assumptions that, as Frege did, tend to simplify the general picture of the world at hands as much as possible. As such, the only way to tackle the formal treatment of vagueness is to enrich the ontological bases of contemporary mathematics. Such requirement is also emerging in the foundations of Computer Science [4], Mathematics and Logic [11] as well as in a more general view of Information management [15]. In this tangled web of demanding conceptual questions and complex technical problems fuzziness plays a specific role by providing computationally affordable quantitative models and conceptually enlarging the horizon of the ingredients used [28, 29, 30].

In our view the problems of vagueness cannot fully be dealt within the horizon of fuzzy set theory, but by comparing their aptitude to be solved by fuzzy and traditional methodologies [31] we can make a step forward to better understand the more general terms of the question.

\section{References}

[1] S. Termini,Vagueness in Scientfic Theories, in in M. G. Singh editor, Systems and Control Encyclopedia, Pergamon Press, Oxford, 4993-4996, 1988.
[2] S. Termini, On some vagaries of vagueness and information, Annals of Mathematics and Artificial Intelligence 35: 343-355, 2002.

[3] H. J. Skala, S. Termini and E. Trillas editors, $A s-$ pects of Vagueness, Kluwer Academic Publishers, 1984.

[4] M.J. Beeson, Towards a Computation System Based on Set Theory, Theoretical Computer Science, 60, 297-340, 1988.

[5] S. Termini, Aspects of Vagueness and Some Problems of their Formalization, in [12], 205-230, 1984.

[6] J. van Heijenoort,"Frege and Vagueness", in Selected Essays, Bibliopolis, 18, 85-97, 1985.

[7] R. Parikh, The problem of Vague Predicates (preprint, 1977) published in Logic, Language and Method (Boston Studies in the Philosophy of Science) R. S. Cohen and M. Wartofsky editors, Reidel, 241-261, 1982.

[8] G. Ludwig, Imprecision in Physics, in A. Hartkamper and H. J. Schmidt editors, Structure and Approximation in Physical Theories, Plenum Press, New York, 7-19, 1981.

[9] L. G. Parratt, Probability and Experimental Errors in Science, J.Wiley and Sons, New York, 1961.

[10] R. Seising, The Fuzzification of Systems, Springer, Berlin, 2007.

[11] L. Montagnini, Looking for a 'scientific' social science: the Macy Conferences on Cybernetics in Bateson's itinerary, Kybernetes 26-7/8, 10121021, 2007.

[12] R. R. Yager, S. Ovchinnikov, R. M. Tong and H.T. Nguyen editors, Fuzzy sets and applications: selected papers by L. A. Zadeh, J. Wiley and Sons, New York, 1987.

[13] L. A. Zadeh, Fuzzy Sets, Information and Control, 8, 338-353, 1965.

[14] R. Seising, On the absence of strict boundariesVagueness, haziness, and fuzziness in philosophy, science, and medicine, Applied Soft Computing 8, 1232-1242, 2008.

[15] A. Pultr, Fuzziness and Fuzzy Equality, Reprinted from Commemtationes Mathematicae Universitatis Carolinae, 23, 2, 1984

[16] M. Black, Vagueness, Philosophy of Science 4, 427-455, 1937.

[17] D. Miller, The accuracy of predictions, Synthese, 30, pp. 159-191, 1975.

[18] D. Miller,Critic al Rationalism. A Restatement and Defense, Open Court, (chapter 11), 1994.

[19] J. Bečvář, Notes on Vagueness and Mathematics, in H. J. Skala, S. Termini and E. Trillas editors, Aspects of Vagueness, Kluwer Academic Publishers., 1-11, 1984.

[20] G. Sambin, Steps towards a dynamic constructivism, in P. Gardenfors, K. Kijania-Placek and J. Wolensky (eds), Proceedings of the XI Internat ional Congress of Logic, Methodology and Philosophy of Science, Krakow, 1999.

[21] P. Vopenka, Mathematics in the Alternative Set Theory, Teubner Verlagsgesellschaft, Leipzig, 1979. 
[22] A. Sochor (1984), The Alternative Set Theory and Its Approach to Cantor's Set Theory, in H. J. Skala, S. Termini and E. Trillas editors, Aspects of Vagueness, Kluwer Academic Publishers, 161-203, 1984.

[23] R. Seising editor, Views on Fuzzy Sets and Systems from Different Perspectives, Springer, Berlin, 2009.

[24] S. Termini, Concepts, Theories, and Applications: the role of "experimentation" for formalizing new ideas along innovative avenues, in: E. Trillas, P.

Bonissone, L. Magdalena and J. Kacprycz editors, Volume in honour of Abe Mamdani, a volume in Physica-Verlag Series 'Studies in Fuzziness and Soft Computing' (in publishing).

[25] J.M. Terricabras, E. Trillas, Some remarks on vague predicates, Theoria $10,1-12,1988$.

[26] J. Beall, Vague intensions: A modest marriage proposal, in Cuts and Clouds, 1, 187-200, 2010.

[27] M. E. Tabacchi and S. Termini, Birkhoff's aesthetics, Arnheim's entropy. Some remarks on complexity and fuzzy entropy in arts, submitted

[28] S. Termini, On Explicandum versus Explicatum: A few elementary remarks on the birth of innovative notions in Fuzzy Set Theory (and Soft Computing). In: Seising, R., Sanz, V. editors, Soft Computing in Humanities and Social Sciences, volume I, Springer series "Studies in Fuzziness and Soft Computing" (in publishing).

[29] S. Termini and M. E. Tabacchi, Fuzzy Set Theory as a methodological brigde between hard and human sciences, submitted

[30] S. Termini, Do Uncertainty and Fuzziness present themselves (and behave) in the same way in Hard and Human Sciences? In: E. Hullermeier, R. Kruse, and F. Hoffmann editors: IPMU 2010, Part II, CCIS 81, Springer-Verlag Berlin Heidelberg, 334-343, 2010.

[31] S. Termini, On some "family resemblances" of Fuzzy Set Theory and Human Sciences. In: Seising, R., Sanz, V. editors, Soft Computing in Humanities and Social Sciences, volume I, Springer series "Studies in Fuzziness and Soft Computing" (in publishing). 